To appear in the Astrophysical Journal, Feb. 10, 1996.

\title{
The Connection between Submillimeter Continuum Flux and Binary Separation in Young Binaries: Evidence of Interaction between Stars and Disks
}

\author{
Eric L. N. Jensen ${ }^{1}$ and Robert D. Mathieu ${ }^{2}$ \\ Department of Astronomy, University of Wisconsin-Madison, Madison, WI 53706 \\ and \\ Gary A. Fuller ${ }^{3}$ \\ NRAO, Edgemont Road, Charlottesville, VA 22903
}

\begin{abstract}
We present $800 \mu \mathrm{m}$ continuum photometry of pre-main-sequence binary stars with projected separations $a_{p}<150$ AU in the Scorpius-Ophiuchus star-forming region. Combining our observations with published $1300 \mu \mathrm{m}$ continuum photometry from André \& Montmerle (1994), we find that binaries in Sco-Oph with $1<a_{p}<50-100$ AU have lower submillimeter continuum fluxes than wider binaries or single stars, as previously found for Taurus-Auriga binaries. The wide binaries and single stars have indistinguishable submillimeter flux distributions. When the Sco-Oph and Tau-Aur samples are combined, this dependence of submillimeter flux on binary separation is detected with a confidence level of greater than $99 \%$. Thus, binary companions with separations less than 50-100 AU significantly influence the nature of associated disks.

We have explored the hypothesis that the reduction in submillimeter flux is the result of gaps cleared in 100-AU disks by companions. Gap clearing produces the qualitative dependence of submillimeter flux on binary separation, and a simple model suggests that large gaps in disks with surface densities
\end{abstract}

\footnotetext{
${ }^{1}$ E-mail: jensen@astro.wisc.edu

${ }^{2}$ E-mail: mathieu@astro.wisc.edu

${ }^{3}$ Previously at NRAO, 949 N. Cherry Ave., Campus Building 65, Tucson, AZ 85721. E-mail: gfuller@nrao.edu. The National Radio Astronomy Observatory is operated by Associated Universities, Inc., under a cooperative agreement with the National Science Foundation.
} 
typical of wide-binary or single-star disks can reduce submillimeter fluxes to levels consistent with the observed limits. This model shows that the present submillimeter flux upper limits do not necessarily imply a large reduction in disk surface densities.

Two-thirds of the pre-main-sequence binaries were detected by IRAS at 60 $\mu \mathrm{m}$, showing that most binaries have at least one circumstellar disk. We have used these fluxes to place lower limits of $10^{-5} M_{\odot}$ on circumstellar disk masses. Similarly, the $60 \mu \mathrm{m}$ fluxes indicate that the circumstellar disk surface densities are no more than two orders of magnitude smaller than those of typical disks around single stars.

Our upper limits on submillimeter fluxes place upper limits of $0.005 M_{\odot}$ on circumbinary disk masses. Thus massive circumbinary disks (such as that found around GG Tau) are rare among binaries with projected separations between a few AU and 100 AU. Circumbinary disks are found around some binaries with separations less than a few AU.

Subject headings: accretion, accretion disks — binaries: visual — infrared: stars — stars: formation — stars: pre-main-sequence

\section{Introduction}

It has been known for some time that a majority of main-sequence stars are in binary systems (e.g. Abt 1983, Duquennoy \& Mayor 1991). Recently, systematic surveys of pre-main-sequence (PMS) stars have shown that their binary frequency is at least as high as, and perhaps significantly higher than, that of main-sequence stars (Mathieu, Walter, \& Myers 1989, Simon et al. 1992, 1995, Ghez, Neugebauer, \& Matthews 1993, Leinert et al. 1993, Reipurth \& Zinnecker 1993). Thus, the most likely outcome of the star formation process is a binary system and understanding binary formation is vital to understanding star formation.

Infrared and millimeter observations have shown that many young stars have excess long-wavelength emission, indicative of circumstellar material. Although this material is unresolved in most observations, there is mounting evidence that in most cases it lies in circumstellar or circumbinary disks (see e.g. Beckwith \& Sargent 1993, Basri \& Bertout 1993, and Sargent 1995 for reviews). Indeed, much of a star's mass may be built up by 
accretion of material through these disks. Thus, disks provide a fossil record of the star formation process, and study of disk properties may reveal clues about star formation.

Observations suggest that the structure of a disk is affected by the presence of an embedded binary. Beckwith et al. (1990, hereafter BSCG) measured $1300 \mu \mathrm{m}$ continuum emission from 86 PMS stars in Taurus-Auriga (Tau-Aur) to search for disks. They found that very few of the binaries in their sample with projected separations less than $100 \mathrm{AU}$ had detectable $1300 \mu \mathrm{m}$ emission while strong emission was common in wider binaries (see also Beckwith \& Sargent 1993). Jensen, Mathieu, \& Fuller (1994, hereafter Paper I) used a larger sample of binaries in Tau-Aur and more sensitive $800 \mu \mathrm{m}$ observations of the systems with the smallest projected separations to further explore this result. We found that the fluxes from binaries with projected separations between 1 and 50-100 AU were significantly lower than those from wider binaries or single stars. On the other hand, the flux distributions of the two latter groups are statistically indistinguishable. Osterloh \& Beckwith (1995) made additional $1300 \mu \mathrm{m}$ observations of PMS stars in Tau-Aur and did detect some close binaries. Nonetheless, they found the difference in flux distributions between close binaries and wide binaries or single stars to remain at a statistically significant level.

There are a variety of theoretical predictions about the influence of binaries on their associated disks. A binary companion embedded in a disk is expected to rapidly clear a gap, isolating distinct circumstellar and circumbinary disks. Such a gap may inhibit the transfer of material from circumbinary to circumstellar disks (e.g. Artymowicz et al. 1991, but see Artymowicz \& Lubow 1994). If so, continued accretion from the circumstellar disks onto the stars may exhaust the reservoir of circumstellar disk material more quickly than in single stars (Clarke 1992). Indeed binary companions may accelerate the depletion. Ostriker, Shu, \& Adams (1992) found that for separations less than 100 AU a companion can excite density waves in a circumstellar disk, causing an enhanced accretion rate onto the central star. At the same time, the inhibition of accretion could increase the lifetimes of circumbinary disks (Clarke 1992). Pringle (1991) found that the transfer of angular momentum from the binary orbit to the circumbinary disk pushes disk material to larger radii, resulting in a reduction in surface density and increase in size of the circumbinary disk. We note that most of these predictions remain largely untested by current observations.

We have undertaken a submillimeter survey of young binaries in the Scorpius-Ophiuchus (Sco-Oph) star-forming region. In this paper, we present $800 \mu \mathrm{m}$ observations of most known Sco-Oph PMS binaries with projected separations $a_{p}$ less than 150 AU. We combine these data with other Sco-Oph submillimeter data from André \& Montmerle (1994) and find a dependence of submillimeter flux on binary separation in a sample that is 
independent of that used by BSCG, Paper I, or Osterloh \& Beckwith (1995). We compare the submillimeter data from Sco-Oph and Tau-Aur with submillimeter fluxes predicted from models of disks with gaps and show that gap clearing alone may be sufficient to explain the low submillimeter fluxes from the close binaries. Most PMS binaries at all separations were detected at $60 \mu \mathrm{m}$ by IRAS, indicating the presence of circumstellar disks. We derive lower limits on circumstellar disk masses from the $60 \mu \mathrm{m}$ emission. Finally, we use submillimeter fluxes to place upper limits of $0.005 M_{\odot}$ on circumbinary disk masses for typical binaries with $a_{p}$ between a few $\mathrm{AU}$ and $100 \mathrm{AU}$.

\section{Observations}

The target binaries (see \$3.) were observed using the James Clerk Maxwell Telescope (JCMT)f on 1992 February 24-27, 1992 March 2-4, and 1993 February 26 - March 1. The JCMT facility ${ }^{3} \mathrm{He}$ cooled bolometer, UKT14, was used for the observations. All observations were made using a 65 -mm aperture which resulted in a $\sim 19^{\prime \prime}$ beam width (FWHM) at all wavelengths. All the observations were made using standard chopping and beam switching with synchronous detection of the signal. The secondary chopper was switched $60^{\prime \prime}$ in azimuth at a rate of $7.8 \mathrm{~Hz}$.

During the 1992 observing sessions the atmosphere was usually stable and transparent. Measurements from a tipping radiometer indicated zenith optical depths of typically 0.03 to 0.04 at $225 \mathrm{GHz}$. The weather during the 1993 run was very stable and the atmosphere transparent. The zenith optical depth at $225 \mathrm{GHz}$ was approximately constant at $\sim 0.08$ during the first night and between 0.04 and 0.05 for the remaining nights. During both runs standard sources of known flux were observed every 15 to 30 minutes in order to monitor the sky opacity. The pointing of the telescope was also checked and corrected regularly during the observations by making five-point measurements on bright sources. Typically the actual pointing was found to be within $\sim 3^{\prime \prime}$ of the nominal pointing. Very occasional shifts as large as $6^{\prime \prime}$ were seen.

Our survey observations were made at $800 \mu \mathrm{m}$. Because thermal dust emission decreases with wavelength from 800 to $1300 \mu \mathrm{m}$ more quickly than the atmospheric transmission improves (in good weather), at the JCMT $800 \mu \mathrm{m}$ observations are more

\footnotetext{
${ }^{4}$ The James Clerk Maxwell Telescope is operated by the Royal Observatories on behalf of the Particle Physics and Astronomy Research Council of the United Kingdom, the Netherlands Organization for Scientific Research, and the National Research Council of Canada.
} 
sensitive for detecting weak dust emission. In addition, non-thermal emission (e.g. free-free or gyrosynchrotron) increases in strength with increasing wavelength, and thus $800 \mu \mathrm{m}$ observations are less likely to be contaminated by this non-disk emission.

The program sources were initially observed for $\sim 1000 \mathrm{~s}$ at $800 \mu \mathrm{m}$. This resulted in a typical $1 \sigma$ noise level of 15-20 mJy. Most sources were observed twice, particularly those for which the noise level of the first observation was higher than the typical level of sources which were marginally detected. Observations at $1100 \mu \mathrm{m}$ and $450 \mu \mathrm{m}$, and occasionally $350 \mu \mathrm{m}$, were made of sources which were strong detections at $800 \mu \mathrm{m}$.

Observations of the standard sources at different airmasses were used to determine the telescope sensitivity at $1100 \mu \mathrm{m}, 800 \mu \mathrm{m}, 450 \mu \mathrm{m}$, and $350 \mu \mathrm{m}$. Given the telescope sensitivity, the standard source observations provided an estimate of the sky opacity as a function of time throughout each night. For both observing sessions, the variation of the sky opacity with time derived from these observations very closely tracked the opacity derived from the $225 \mathrm{GHz}$ radiometer. The program sources were calibrated using the mean sky opacity derived from standard sources observed before and after the target source. The scatter in the zenith opacity about a linear fit versus time for each night has been used as an estimate of the uncertainty in the flux calibration.

Each observation consisted of a series of 10-second samples. To remove noise spikes from the data, the mean of the samples in each integration was calculated and those samples which deviated from the mean by more than three standard deviations were removed and the mean was recalculated. The measured fluxes or $3 \sigma$ upper limits are given in Table 1 . The quoted uncertainties are a combination of the flux calibration uncertainty discussed above and the photon noise derived from the standard deviation of the individual 10-second samples within each observation.

\section{Sample selection}

We chose binaries for our sample from among members of the Upper Scorpius subgroup of the Sco OB2 association and from the $\rho$ Oph cloud complex. These stars lie within the approximate boundaries of right ascension $15^{\mathrm{h}} 10^{\mathrm{m}}-16^{\mathrm{h}} 40^{\mathrm{m}}$ and declination $-5^{\circ}--35^{\circ}$ (Blauuw 1978). We will refer to this area as "Sco-Oph". We adopt distances of 125 pc for the $\rho$ Oph clouds and 160 pc for Upper Sco (de Geus, de Zeeuw, \& Lub 1989).

Surveys which have searched for binaries in Sco-Oph include those of Mathieu et al. (1989), Ghez et al. (1993), Reipurth \& Zinnecker (1993), and Simon et al. (1992, 1995). 
These surveys vary greatly in their target lists and the range of binary separations to which they are sensitive. The characteristics of the surveys are summarized in Table 2. In addition, several PMS binaries have been found by observations that were not part of surveys. AK Sco was discovered to be a spectroscopic binary by Andersen et al. (1989). WL 2 and WL 20 were both found to be binaries by infrared imaging (Rieke, Ashok, \& Boyle 1989, Barsony et al. 1989). VV Sco was noted as a visual binary by Gregorio-Hetem et al. (1992). DoAr 51 was found to be a binary by Koresko (1995) using speckle interferometry.

Currently only spectroscopic observations can detect binaries with separations less than $\sim 1$ AU at the distance of Sco-Oph. All but one (AK Sco) of the known spectroscopic binaries in Sco-Oph were found in a radial-velocity survey of x-ray selected stars in Sco-Oph (Mathieu et al. 1989). Independent of multiplicity, most of the x-ray selected stars surveyed by Mathieu et al. (1989) do not have infrared excesses or strong $\mathrm{H} \alpha$ emission (Walter et al. 1994), the classical signatures of disks. Thus the binaries discovered in that survey may not be representative of PMS spectroscopic binaries in general, particularly in terms of the disk properties with which we are concerned here.

In contrast to the spectroscopic observations of Mathieu et al. (1989), the other surveys for binaries in Sco-Oph have drawn their target lists primarily from lists of $\mathrm{H} \alpha$ - and infrared-selected PMS stars. Ghez et al. (1993) and Simon et al. (1995) also include some $\mathrm{x}$-ray selected PMS stars in their target lists. The targets of these surveys were not initially discovered to be young stars using a uniform set of selection criteria, and thus the combined sample of Sco-Oph binaries from these surveys is not a well-defined, uniformly-selected PMS binary sample. However, the diversity of targets of these surveys reflects the variety of techniques that have been used to search for young stars. Thus, while the sample of binaries discovered by these surveys may not be uniformly defined, it should be reasonably representative of the known population of PMS stars in Sco-Oph in general.

Because the spectroscopic binaries are drawn from a distinctly different sample of PMS stars than are the other binaries, in this paper we limit our analysis to binaries with projected separations greater than 1 AU. This sample should be neither more nor less biased toward the presence of disks than the known Sco-Oph PMS population as a whole. For these binaries, we present a quantitative analysis of the distribution of submillimeter fluxes with projected separation and discuss the implications of our data for binary-disk interactions. We will discuss the submillimeter fluxes and spectral energy distributions of binaries with projected separations less than $1 \mathrm{AU}$ in a later paper.

Simon et al. $(1992,1995)$ argue that for separations less than 10", contamination of the binary sample from chance superposition of stars should be negligible. Thus, we adopt this as the separation upper bound for our sample. We have taken projected separations or 
projected semimajor axes for Sco-Oph binaries from the binary surveys discussed above.

The other primary requirement for inclusion in our sample is availability of an 800 $\mu \mathrm{m}$ or $1300 \mu \mathrm{m}$ flux measurement. (For convenience, we will refer to either of these as "submillimeter fluxes.") Submillimeter fluxes or flux limits are from this work and André \& Montmerle (1994, hereafter AM), which together cover about $90 \%$ of the currently known PMS binaries in Sco-Oph.

Our goal is to explore the influence of multiplicity on the properties of circumstellar and circumbinary disks. In order to avoid confusion introduced by other factors that may also influence submillimeter emission, we have further constrained our sample. Our sample is limited to PMS stars with spectral types of $\mathrm{F}$ and later so as to reduce the range of stellar mass, luminosity, and temperature. We have also excluded Class I sources as defined in the infrared classification scheme of Lada (1987). Terebey, Chandler, \& André (1993) suggest that a significant fraction of the $1300 \mu \mathrm{m}$ emission from Class I sources arises in an envelope rather than a disk; this is consistent with the fact that Class I sources have significantly higher $1300 \mu \mathrm{m}$ fluxes on average than Class II or Class III sources and that Class I sources tend to be extended whereas Class II sources are unresolved (AM). Class I sources are also heavily embedded and presumably younger than Class II or III sources (AM). In fact, the spectral type and infrared class criteria remove only a few systems from our sample.

After applying these selection criteria, we obtain a sample of 30 multiple systems in Sco-Oph. This is comparable to the 42 systems in Tau-Aur studied in Paper I. With the addition of data from Osterloh \& Beckwith (1995), the present Tau-Aur sample includes 55 multiple systems. We also analyze this expanded sample below.

In addition to the binaries, we adopt a sample of single stars for comparison. Single stars provide a "control" sample, revealing submillimeter fluxes produced in the absence of any stellar-mass companion. We take our single-star sample from the targets observed by $\mathrm{AM}$ which are not known to have stellar companions within $10^{\prime \prime}$. We apply the same spectral type and IR class criteria described above. From the $\rho$ Oph region, we adopt only the sources that AM have marked with a "\#" in their Table 1, since these are all confirmed PMS stars which have been observed at a uniform sensitivity. In addition, we adopt the other single PMS stars which AM observed that lie outside $\rho$ Oph but within Sco-Oph. This yields a total of 47 stars.

We note that this definition of our single star sample is less restrictive than in Paper I for the Tau-Aur sample, where we adopted as single only those stars which had been surveyed using speckle interferometry or lunar occultation and found not to have companions. However, the surveys for binaries in Sco-Oph have been less comprehensive 
and less uniform than in Tau-Aur; applying such a criterion to Sco-Oph would yield a prohibitively small sample of 15 stars. Thus, here we adopt as single those stars not known to have companions, whether or not they have been surveyed with high-resolution techniques. To estimate the contamination from unresolved companions in our single-star sample, we note that of 47 stars in the "single" sample, 15 have been surveyed using high-resolution techniques, leaving 32 of unknown multiplicity. Adopting a multiplicity frequency of $50 \%$ (Simon et al. 1995 find $48 \%$ for Tau-Aur and Oph), we then expect $\sim 16$ binaries out of 47 stars in the sample. Wide companions $\left(a_{p} \gtrsim 1^{\prime \prime}\right)$ would be more likely to have been previously discovered, so the contamination is likely to be predominantly close binaries. Thus the expected effect of contamination of the single sample by undetected binaries would be to weaken the statistical significance of any differences between the flux distributions of the close binaries and single stars.

\section{The dependence of submillimeter flux on binary separation}

\subsection{Combining $1300 \mu \mathrm{m}$ and $800 \mu \mathrm{m}$ flux measurements}

The bulk of the submillimeter and millimeter continuum flux measurements of PMS stars in the literature are contained in the surveys of BSCG, Osterloh \& Beckwith (1995) (both including Tau-Aur), and AM (Sco-Oph). All of these surveys were made at a wavelength of $1300 \mu \mathrm{m}$ while our observations were made at $800 \mu \mathrm{m}$. Combining these measurements raises the question of how to compare fluxes measured at $1300 \mu \mathrm{m}$ with those measured at $800 \mu \mathrm{m}$. Because of the existence of a large number of $1300 \mu \mathrm{m}$ flux measurements of PMS stars in the literature, we have chosen to scale our $800 \mu \mathrm{m}$ fluxes or flux limits to $1300 \mu \mathrm{m}$ for comparison with existing data. Submillimeter and millimeter fluxes from dust around PMS stars are dominated by emission on the Rayleigh-Jeans tail of the Planck function, and thus if the emission is optically thick the flux density $F_{\nu}$ should vary with frequency as $\nu^{2}$. If the emission is optically thin and the submillimeter dust opacity varies as $\kappa_{\nu} \propto \nu^{\beta}$, then the flux should vary as $\nu^{2+\beta}$. Since dust opacity is thought to increase with increasing frequency (i.e. $\beta$ is positive; Beckwith \& Sargent 1991, Pollack et al. 1994, Agladze et al. 1994), then the variation of submillimeter and millimeter thermal continuum emission with frequency should be at least as steep as $F_{\nu} \propto \nu^{2}$. In fact, observations of PMS stars at multiple millimeter wavelengths bear this out. Beckwith \& Sargent (1991) observed 25 PMS stars and found that 19 out of 25 have millimeter spectral slopes greater than or equal to $\nu^{2}$ within their $1 \sigma$ uncertainties. 
Thus, for systems with upper limits on their $800 \mu \mathrm{m}$ fluxes we calculate $1300 \mu \mathrm{m}$ upper limits by multiplying the $800 \mu \mathrm{m}$ flux limits by $\left(\nu_{1300} / \nu_{800}\right)^{2}=0.38$. Since the true spectral slope may be steeper than $\nu^{2}$, this scaling provides a conservative but secure upper limit on the $1300 \mu \mathrm{m}$ flux. For systems with upper limits at both 800 and $1300 \mu \mathrm{m}$, the measured $1300 \mu \mathrm{m}$ limit is compared to the $800 \mu \mathrm{m}$ limit scaled to $1300 \mu \mathrm{m}$, and the lower of the two values is used.

One system in our Sco-Oph sample was detected at $800 \mu \mathrm{m}$ but not $1300 \mu \mathrm{m}$. Haro 1-4 was not detected by AM with a $1300-\mu \mathrm{m}$ flux limit of $F_{\nu}<50 \mathrm{mJy}$. This limit is consistent with our $800 \mu \mathrm{m}$ detection of $F_{\nu}=58 \mathrm{mJy}$. To estimate the $1300 \mu \mathrm{m}$ flux of Haro 1-4, we extrapolated the 800- $\mu \mathrm{m}$ flux to $1300 \mu \mathrm{m}$ assuming a spectral slope of $\nu^{2}$, the most common slope found by Beckwith \& Sargent (1991). Nonetheless, the unknown spectral slope leads to an uncertain $1300 \mu \mathrm{m}$ flux. However, tests using different slopes to determine the flux of Haro 1-4, or leaving it out of the sample altogether, show that the effect of this uncertainty on our results is negligible.

A few binary systems do not show the variation of submillimeter flux with wavelength expected for thermal emission from dust. We treat these on a case-by-case basis. V773 Tau was detected at $1300 \mu \mathrm{m}$ by BSCG with a flux of $42 \pm 6 \mathrm{mJy}$ and by Osterloh \& Beckwith (1995) with a flux of $24 \pm 4 \mathrm{mJy}$. However, we did not detect it at $800 \mu \mathrm{m}$ with a $3 \sigma$ upper limit of $29 \mathrm{mJy}$ (Paper I). The apparent variability at $1300 \mu \mathrm{m}$, the $800-1300 \mu \mathrm{m}$ spectral slope much flatter than $\nu^{2}$, and the strong non-thermal centimeter wavelength emission (Phillips, Lonsdale, \& Feigelson 1991) suggest that at least some of the $1300 \mu \mathrm{m}$ flux from V773 Tau is produced by a non-thermal process and is not emission from circumstellar dust. As in Paper I, we proceed on the assumption that the $1300 \mu \mathrm{m}$ emission is non-thermal, and we use the $800 \mu \mathrm{m}$ limit from Paper I to derive an upper limit on the $1300 \mu \mathrm{m}$ disk emission.

Two binaries in Sco-Oph (SR 9 and DoAr 24E) show a similar disagreement of $800 \mu \mathrm{m}$ and $1300 \mu \mathrm{m}$ fluxes. We did not detect SR 9 at $800 \mu \mathrm{m}$ with a $3 \sigma$ upper limit of $28 \mathrm{mJy}$. Scaling this to $1300 \mu \mathrm{m}$ by $\nu^{2}$ yields an upper limit of $11 \mathrm{mJy}$, whereas AM list it as a weak detection $(\sim 4 \sigma)$ at $1300 \mu \mathrm{m}$ with a flux of $15 \mathrm{mJy}$. However, in this case there is no independent evidence of non-thermal emission; SR 9 was undetected at $6 \mathrm{~cm}$ (André et al. 1990). SR 9 may be similar to the few systems found by Beckwith \& Sargent (1991) to have submillimeter spectral slopes slightly flatter than $\nu^{2}$ (i.e. $-1<\beta<0$ ). Because of the lack of other evidence for non-thermal contributions to the millimeter flux and the relatively modest deviation from expected dust-emission spectral slope, for SR 9 we adopt the $1300 \mu \mathrm{m}$ flux measured by AM for our analysis. DoAr 24E, however, shows a larger disagreement between 800 and $1300 \mu \mathrm{m}$ fluxes. We measured a flux of $37 \pm 8 \mathrm{mJy}$ at 800 
$\mu \mathrm{m}$, while AM measured $65 \mathrm{mJy}$ at $1300 \mu \mathrm{m}$. These measurements imply $\beta \approx-3.2$, well outside the range of $\beta$ values found by Beckwith \& Sargent (1991). Reipurth \& Zinnecker (1993) report a $1300 \mu \mathrm{m}$ flux from DoAr 24E of $\sim 20 \mathrm{mJy}$. Although no radio continuum measurements of DoAr 24E are known to us, its unusual submillimeter spectral slope and apparent variability (as in the case of V773 Tau) suggest a non-thermal contribution to its $1300 \mu \mathrm{m}$ flux. Thus, we adopt our $800 \mu \mathrm{m}$ flux scaled to $1300 \mu \mathrm{m}$ by $\nu^{2}$ as the best estimate of the disk contribution to submillimeter emission for the analysis below. Finally, we emphasize that, given the size of our sample, the particular choice of flux for these three systems (or even their inclusion or omission) does not affect the conclusions of our analysis.

\subsection{Assignment of flux in unresolved triple and quadruple systems}

Five systems in our Sco-Oph sample each comprise three or four stars that lie within the JCMT beam: V853 Oph, SR 24, 155913-2233, 162814-2427 (ROX 42C), and 162819-2423 (ROX 43A/B). In the Tau-Aur binary sample, there are six systems which are triples with all components within 10" of each other: HP Tau/G3, V807 Tau, HV Tau, RW Aur, UZ Tau, and UX Tau. In the absence of spatially-resolved flux measurements of the individual components of such higher-order multiple systems, it is unknown whether any detected flux should be associated with the binary, the tertiary, or both. Thus the positions of such systems in a plot of flux vs. projected separation are ambiguous.

For systems that are not detected, we know that neither the close pair nor the wide companion has substantial submillimeter flux; thus the system is reasonably represented by two points, one at each projected separation. For detected systems, the flux could arise from the close pair, the wide companion, or both. For these systems we have also plotted two points, one at each projected separation. For the pair with the larger projected separation, at least one member (whether that member is single or multiple) of the wide pair has detectable submillimeter flux. Thus, there is still detectable flux in the presence of a wide companion which should be represented by a point on the plot at the wider projected separation. For the pair with the closer projected separation, there may be detectable

\footnotetext{
${ }^{5}$ However, a counter argument to this is that a hierarchical quadruple system is quite different from just a wide binary, since this "wide pair" would not be expected to have a large flux if both close binaries cleared their disks. The only quadruple in our sample is 162819-2423 (ROX 43A/B), which is represented by three data points, one at each projected separation in the system. However, the closest pair in the system (the spectroscopic binary 162819-2423S) has a separation smaller than the 1 AU lower bound of our sample. Thus only two points for this system appear on the plots and in the statistical analysis.
} 
submillimeter flux from a close pair, and thus we adopt the conservative approach of putting a point on the plot to represent this possibility. Since we have argued in Paper I that close pairs have smaller fluxes on average than wide pairs, this method of assigning fluxes provides the most rigorous test of our hypothesis.

Two triple systems, SR 24 and UZ Tau, have been treated differently because the wide pair in each has been resolved at millimeter wavelengths. SR $24 \mathrm{~N}$ and S are separated by $6^{\prime \prime}$, and SR $24 \mathrm{~N}$ is a close binary with a projected separation of $0^{\prime \prime} 2$ (Simon et al. 1995). AM mapped the area around SR 24 using an "on-the-fly" mapping technique and a 12 " (FWHM) beam, and they conclude that the detected $1300 \mu \mathrm{m}$ emission arises from an unresolved area around SR 24S, with an upper limit of $30 \mathrm{mJy}$ on emission from SR $24 \mathrm{~N}$, the close binary. They give fluxes of $280 \mathrm{mJy}$ for SR $24 \mathrm{~S}$ and $<30 \mathrm{mJy}$ for SR $24 \mathrm{~N}$, though they note that the latter limit is uncertain due to possible contamination from SR $24 \mathrm{~S}$. This is consistent with the results of Reipurth et al. (1993), who found the total SR 24 flux in a $23^{\prime \prime}$ beam to be $259 \pm 14 \mathrm{mJy}$ at $1300 \mu \mathrm{m}$. We adopt the flux values given by AM for our analysis. For UZ Tau, the wide pair has been resolved at $1300 \mu \mathrm{m}$ in interferometric observations using the Owens Valley Millimeter Array (Jensen, Koerner, \& Mathieu 1995, in preparation). UZ Tau W, the close binary, has a flux of $32 \pm 9 \mathrm{mJy}$ at $1300 \mu \mathrm{m}$, while UZ Tau E has a flux of $137 \pm 28$ mJy. In our analysis we have assigned UZ Tau W its measured $1300 \mu \mathrm{m}$ flux and assigned the combined flux of both components to the wide E-W pair. Because of the availability of observations which resolve the wide pairs, these two systems are not marked as triples in Figures 1 . 4 .

\subsection{The submillimeter flux distribution as a function of binary separation}

Figure 1 shows the $1300 \mu \mathrm{m}$ flux of Sco-Oph binaries plotted as a function of projected binary separation. Filled symbols are detections and open symbols represent $3 \sigma$ upper limits. Squares show measurements at $1300 \mu \mathrm{m}$, while triangles are $800 \mu \mathrm{m}$ measurements scaled to $1300 \mu \mathrm{m}$ as described above. To aid in determining which points may be confused by the presence of higher-order multiplicity, each point representing a pair in an unresolved higher-order multiple system is marked with a " $T$ " to the right of the point. Different pairings from the same system can be associated by having the same flux measurement. (Note that in three instances the systems include a spectroscopic binary with a separation smaller than the limit of the figure.) All fluxes have been scaled to a distance of $140 \mathrm{pc}$, roughly the mean distance between the $125 \mathrm{pc}$ distance to $\rho$ Oph and the $160 \mathrm{pc}$ distance to Upper Sco. 
A dependence of submillimeter flux on projected binary separation is evident in Figure [1. Binaries with projected separations $a_{p}$ less than roughly $100 \mathrm{AU}$ tend to have lower fluxes than wider binaries. Among binaries with $a_{p} \geq 100 \mathrm{AU}, 7$ of 15 are detected, while only 3 of 16 closer systems are detected, and two of these have separations very near 100 AU. More importantly, the close binaries which were not detected have flux upper limits substantially lower than most of the detections among the wider binaries. Clearly the submillimeter flux distribution differs between binaries with separations greater or less than roughly $100 \mathrm{AU}$, with the distribution extending to larger fluxes among the wider binaries.

In order to quantify the dependence of submillimeter flux on binary separation, we applied statistical tests to compare the distributions of flux among different groups of binaries. We used the techniques of survival analysis which allow quantitative analysis of data which include upper limits. We used the software package ASURV Rev. 1.1 (LaValley, Isobe, \& Feigelson 1992) which implements the methods presented in Feigelson \& Nelson (1985). We divided the data into subsets based on projected separation and then compared pairs of these subsets using the Gehan, logrank, Peto-Peto, and Peto-Prentice two-sample tests. These tests yield probabilities that the distributions of flux in the two samples are drawn from the same parent distribution. The various tests are sensitive to different underlying flux distributions; since the true flux distribution for our sample is unknown, we have applied all of the tests and report the spread of the results.

We have divided the sample into close and wide binaries using separations of both 50 AU and $100 \mathrm{AU}$ as in Paper I. The results of the two-sample tests are given in Table o expressed as percentage confidence levels that the flux distributions of the two samples are different. These results support the conclusion reached by visual examination of Figure 1: at the $90-95 \%$ confidence level binaries with projected separations less than or equal to 100 AU do not have the same submillimeter flux distribution as binaries with greater projected separations. When the sample is divided at $50 \mathrm{AU}$, the same result is found with similar confidence levels.

Simon et al. $(1992,1995)$ found that young binaries as a group (i.e. of all separations) have lower submillimeter fluxes on average than young single stars. We have also compared the sample of "single" stars to the known binaries, but we have considered the close and wide binaries separately. The confidence levels at which the distributions of binaries and single stars differ are given in Table 3. The close binaries have a different flux distribution from the single stars at confidence levels around 95\%. In contrast, the fluxes from the wide binaries and single stars are consistent with being drawn from the same distribution.

We have applied the same two-sample tests discussed above to the PMS binaries and single stars in the Taurus-Auriga star-forming region. These results are also given in 
Table 3, and the Taurus-Auriga binaries are plotted in Figure 2. As expected, the results are the same as from similar previous analyses (Paper I; Osterloh \& Beckwith 1995): the submillimeter flux distribution of the close binaries is distinct from that of the wide binaries at high confidence levels. Thus, the same variation of submillimeter flux with binary separation is seen in two independent samples of PMS stars. Finally, we combine the Sco-Oph and Tau-Aur samples (Figure 3), obtaining confidence levels greater than $99 \%$ that the submillimeter flux distributions of close and wide binaries, and of close binaries and single stars, are distinct.

Hence we conclude that at a statistically secure level the submillimeter flux distribution among binaries with separations between $1 \mathrm{AU}$ and 50-100 AU is different than that of wider binaries or single stars. The sense of the difference is that submillimeter emission is weaker in close binaries.

The binary separation at which the transition occurs is not well defined but appears to be roughly $50 \mathrm{AU}$ to $100 \mathrm{AU}$. This separation is comparable to the radius typically derived for dust disks around PMS stars (e.g. Beckwith \& Sargent 1993). As pointed out by Osterloh \& Beckwith (1995), this transition separation strongly suggests that the emitting material does lie in disks in most cases. If the material were in extended envelopes (e.g. Terebey et al. 1993), binaries at this separation would not be expected to influence the submillimeter emission.

The low submillimeter fluxes from close binaries are not due to age; the closer binaries have the same distribution of ages as the wider binaries or single stars. In addition, within the range of ages (roughly $10^{5}-10^{7} \mathrm{yr}$ ) in our sample, we find no dependence of submillimeter flux on age of the system for the wide binaries.

\section{Interpreting the relationship between disk emission and binary separation}

\subsection{Resonant clearing of gaps in disks}

The presence of a stellar companion at a separation less than the disk radius must influence the spatial distribution of the disk material. Theoretical calculations and numerical simulations show that a binary embedded within a disk will rapidly clear a region on the size scale of the binary separation, thus isolating circumstellar and circumbinary disks (see e.g. Lin \& Papaloizou 1993 for a review). The extent of the region cleared depends on the details of the given system, specifically the binary mass ratio, orbital eccentricity, 
and disk viscosity. Artymowicz \& Lubow (1994) find that circumstellar disks will have outer radii of less than half the binary semi-major axis, and circumbinary disks will have inner radii of greater than roughly twice the binary semi-major axis.

Disk clearing of this type creates a natural link between binary separation and the submillimeter fluxes from binary systems which is very similar to that observed. Assume that disks have a certain characteristic outer radius $R_{d}$. For the purposes of this discussion, this radius could be either a physical limit on the extent of disk material or an effective radius inside which most of the disk's submillimeter emission originates (even though there may be additional cold or low-density material outside it). In binary systems with separations greater than a few times $R_{d}$, each component of the system could have a circumstellar disk that is relatively undisturbed by its companion, and the submillimeter emission from such a system would be comparable to that from a single star with a circumstellar disk. In systems with separations much less than $R_{d}$ only a small hole at the center of the circumbinary disk will be cleared, leaving a circumbinary disk which could be similar in extent and emission to disks around single stars. Binaries with separations somewhat less than $R_{d}$, however, will clear regions whose extent represents a large fraction of the surface area of an undisturbed disk. This reduction in emitting surface area will result in reduced submillimeter fluxes, as observed.

In order to investigate the effect of gap clearing on submillimeter flux in a more quantitative way, we have introduced gaps into a simple disk model. Following a standard approach in modeling infrared and submillimeter disk emission (e.g. Adams, Lada, \& Shu 1988, Adams, Emerson, \& Fuller 1990, BSCG), we use a geometrically thin disk with power-law temperature and surface-density distributions:

$$
\begin{gathered}
T(r)=T_{1}(r /(1 \mathrm{AU}))^{-q}, \\
\Sigma(r)=\Sigma_{0}\left(r / r_{0}\right)^{-p} .
\end{gathered}
$$

The emission from such a disk is then given by

$$
F_{\nu}=\frac{\cos \theta}{D^{2}} \int_{r_{0}}^{R_{d}} B_{\nu}[T(r)]\left(1-e^{-\tau_{\nu}(r)}\right) 2 \pi r d r
$$

where $D$ is the distance from the observer to the system, $\theta$ is the angle between the line of sight and the normal to the disk plane, and $\tau_{\nu}$ is the line-of-sight optical depth through the disk, related to the mass opacity $\kappa_{\nu}$ by

$$
\tau_{\nu}(r)=\frac{\kappa_{\nu} \Sigma(r)}{\cos \theta}
$$

To simulate disk clearing by a binary companion, we made a simple modification to this disk model. For each model binary-disk system, we set the disk surface density (and 
thus the emission) equal to zero between radii $r_{\text {in }}$ and $r_{\text {out }}$, designating the inner and outer edges of the gap:

$$
\Sigma(r)= \begin{cases}0, & \text { if } r_{\text {in }}<r<r_{\text {out }} \\ \Sigma_{0}\left(r / r_{0}\right)^{-p}, & \text { otherwise }\end{cases}
$$

These radii are determined by the parameters of a given binary system, especially the binary semi-major axis $a$. We considered two different cases. In the first, $r_{i n}=0.4 a$ and $r_{\text {out }}=1.8 a$. This is the clearing expected for a binary with a circular orbit, and the minimum clearing expected for any binary system (Artymowicz \& Lubow 1994). In the second case, $r_{i n}=0.2 a$ and $r_{\text {out }}=3 a$. This level of clearing is expected for a binary with an orbital eccentricity of 0.4 , roughly the mean eccentricity for pre-main-sequence and main-sequence binaries with known orbits (Mathieu 1994, Duquennoy \& Mayor 1991).円

For a given set of disk parameters $\left(M_{d}, q, T_{1} ; p=1.5, R_{d}=100\right.$ AU) we calculated the expected submillimeter flux from binaries having a range of separations from $0.01 \mathrm{AU}$ to $2000 \mathrm{AU}$, spanning the observed range of separations in our sample. We then calculated models with a range of inclination angles and averaged their fluxes based on the frequency of occurrence of a given value of $\theta$ in a random distribution of disk inclinations. The result for one particular disk model is shown in Figure 1 . The model is superimposed on the combined submillimeter data from the Sco-Oph and Tau-Aur regions, with all fluxes scaled to a distance of $140 \mathrm{pc}$. The solid line shows the expected submillimeter flux as a function of separation if gaps extend from $0.2 a$ to $3 a$ (eccentric orbits), and the dashed line shows the expected flux if gaps extend from $0.4 a$ to $1.8 a$ (circular orbits).

As expected, the gap-clearing model shows the submillimeter fluxes of the closest and widest binaries to be unaffected by gap clearing, while binaries of intermediate separations have lower fluxes. For the particular model shown the reduction in flux occurs for binaries with separations between roughly $1 \mathrm{AU}$ and $300 \mathrm{AU}$, with a minimum flux around $25 \mathrm{AU}$. Thus our simple model for gap clearing reproduces the qualitative features of the observed submillimeter flux dependence on binary separation.

If the gaps are of the size expected for eccentric binary systems, the reduction of submillimeter flux due to gaps can be as much as a factor of 15 . This is comparable to the difference between typical detected fluxes among wide binaries and the available

\footnotetext{
${ }^{6}$ These clearing radii also depend on the disk viscosity and the binary mass ratio. The numbers quoted here are for a Reynolds number of $10^{5}$ and a binary mass ratio of 7:3. However, changing these values within reasonably expected bounds does not greatly affect the expected disk clearing. For a range of Reynolds numbers from $10^{4}$ to $10^{6}$ and eccentricity of 0.4 , the inner gap edge ranges from $0.19 a$ to $0.25 a$ and the outer gap edge from $2.7 a$ to $3.1 a$ (Artymowicz \& Lubow 1994). We adopt the numbers above as representative of an "average" binary.
} 
upper limits for the close binaries. The model shown in Figure 4 provides a specific case for quantitative discussion. This model has $M_{d}=0.05 M_{\odot}, q=0.65$, and $T_{1}=125 \mathrm{~K}$ and was chosen to match typical flux levels among detected wide binaries. The minimum submillimeter flux in this particular model is a factor of nine less than the flux of the disk with no gap. The dashed line (circular binary) lies well above most of the observed flux upper limits between 1 and $100 \mathrm{AU}$ and thus is not consistent with the observations. However, most binaries have eccentric orbits. The solid line $(e=0.4)$ passes through the upper limits for binaries with separations around $25 \mathrm{AU}$ and exceeds the typical upper limits for binaries of somewhat larger and smaller separations by a factor of two to three at most.

The essential conclusion to be drawn from this simple model is that the lack of detected submillimeter emission among the closer binaries does not necessarily imply that disk material is not present, nor that disk surface densities are much lower than found among wide binaries or single stars. The large reduction in emitting surface area due to gap clearing can in and of itself reduce flux to levels comparable to many of the present upper limits. Thus, while it is clear from these submillimeter data that binary companions with separations less than $100 \mathrm{AU}$ significantly influence the nature of associated disks, it remains an outstanding question to what extent circumstellar and circumbinary disks are present in these binaries. We return to these issues in Sections 5.2. and 5.3.

Given the presence of a companion, the physical conditions of the disk(s) in our simple model are certainly oversimplified, and thus a detailed comparison of the model with observations is likely not merited. Nonetheless, we note that the model appears less successful for binaries with separations between $1 \mathrm{AU}$ and $10 \mathrm{AU}$. In particular, the model flux is symmetric in $\log \left(a_{p}\right)$ around binary separations of a few tens of $\mathrm{AU}$, while in fact submillimeter fluxes remain low at separations less than $10 \mathrm{AU}$. Unfortunately, the sample size in this separation range is small; the two-sample tests give probabilities of only $78-90 \%$ that the submillimeter fluxes of binaries with $1<a_{p}<10 \mathrm{AU}$ are drawn from a different distribution than the fluxes of binaries with $a_{p}>100$ AU. Furthermore, most of the binaries with $1<a_{p}<10 \mathrm{AU}$ were discovered via lunar occultation techniques, so that the measured separation is a projection against the lunar limb. As such, many of these binaries may have wider separations projected on the sky than those shown in Figure 4 . Thus, while there is a suggestion that there is a larger reduction in flux among the closest binaries than predicted by the model, the case is not strong with the present data.

If the reduction in submillimeter flux does extend to separations as small as $1 \mathrm{AU}$, this would be difficult to reproduce with our simple annular gap model. We note that the flux predicted by the model for binaries with separations of less than $10 \mathrm{AU}$ is largely from 
circumbinary disks. Indeed for binaries with separations of only a few AU the surface areas of circumstellar disks are too small to produce detectable submillimeter emission. Hence weak submillimeter emission from such binaries would suggest lower mass, temperature, and/or dust opacity of circumbinary material.

Finally, we note that the most submillimeter-luminous binaries, such as GG Tau, T Tau, and AS 205, cannot be easily incorporated into this simple picture. GG Tau in particular has a projected separation of $40 \mathrm{AU}$ and yet is the most luminous binary in the Tau-Aur and Sco-Oph regions. Furthermore, interferometric observations at millimeter wavelengths reveal a circumbinary disk with a radial extent of at least $800 \mathrm{AU}$ (Dutrey, Guilloteau, \& Simon 1994). The millimeter emission also shows a central depression which Dutrey et al. attribute to a central cavity of radius 180 AU. They attribute this cavity to a clearing process similar to that invoked here for gaps, but clearly this system is substantially different from both our standard model for a 40 AU binary and from other binaries with projected separations of tens of AU.

\subsubsection{Effect of disk parameters on the flux-separation relation}

The model shown in Figure 1 is only one realization of a large range of physical conditions found in disks. Therefore it is useful to establish whether other combinations of model parameters can better reproduce the observed variation of flux with separation. In Figure 5 we explore the influence of disk parameters on the distribution of submillimeter flux with binary separation. In panels a, b, and c, one of the disk parameters $T_{1}, q$, and $M_{d}$ is varied while the others are held fixed. In panel d, all three disk parameters are held fixed while the wavelength at which the flux is calculated is varied.

One general trend that can be seen in these figures is that changes in disk properties which give lower submillimeter fluxes also tend to decrease the binary separation corresponding to the minimum flux. This is because the contribution of the hotter, more optically thick inner regions of the disk contribute a larger percentage of the total flux when the disk luminosity is low. For more luminous disks whose outer regions are hotter (through higher $T_{1}$ or lower $q$ ) or more optically thick (through higher $M_{d}$ ), the contribution of the outer disk to the total flux is significant. In such disks gap clearing in the outer regions has a larger effect on the total submillimeter flux.

A discontinuous change in slope can be seen in some of the models in Figure 5 (as well as in Figure (1). This is due to the assumption in the models that the disks have a sharp 
outer radius $R_{d}$, here taken to be $100 \mathrm{AU}$. The slope discontinuity occurs when the outer edge of the gap $r_{\text {out }}$ reaches the outer edge of the disk. Increasing $R_{d}$ would increase the separation that gives the minimum flux for a few of the models shown in Figure 5, though most would be unchanged.

Interestingly, the choice of $1300 \mu \mathrm{m}$ or $800 \mu \mathrm{m}$ as an observing wavelength makes little difference in the binary separation producing the minimum flux (Figure 5d). This is due to the interplay of two opposing factors. Shorter-wavelength emission is more sensitive to disk material at higher temperatures, suggesting that $800 \mu \mathrm{m}$ emission would tend to probe gaps at smaller disk radii than $1300 \mu \mathrm{m}$ emission. However, for emission in the Rayleigh limit the tradeoff between increasing surface area and decreasing optical depth with radius tends to emphasize the emission from the region in the disk near $\tau_{\nu}=1$ (BSCG). Because dust opacity increases with frequency, $\tau_{\nu}=1$ lies at a larger radius for $800 \mu \mathrm{m}$ than for $1300 \mu \mathrm{m}$. These two effects largely cancel each other and emission at both wavelengths is sensitive to roughly the same disk radii. However, $60 \mu \mathrm{m}$ flux is sensitive to smaller disk radii because much of the disk is too cold to produce appreciable $60 \mu \mathrm{m}$ emission.

We draw two main conclusions from this exploration of model parameter space. First, under no circumstances does gap clearing at the level expected for binaries with circular orbits produce a reduction in flux that is comparable to that observed. Second, no combination of model parameters does significantly better than that in Figure 1 at reproducing the depth, breadth, and location of the reduction in submillimeter fluxes observed among the close binaries.

\subsubsection{Estimates of disk masses}

Gap clearing may affect not only the distribution of submillimeter flux with separation, but also the disk mass derived for a given system based on its submillimeter flux. Submillimeter fluxes have commonly been used to infer disk masses by adopting a model for a continuous disk, deriving the disk temperature distribution from infrared data, and then adjusting the disk mass until the model flux agrees with the observed submillimeter flux at one or more wavelengths (Adams et al. 1990, BSCG, AM, Osterloh \& Beckwith 1995). However, if binaries clear gaps in their disks, the geometry of the emitting material is significantly different than that assumed in conventional disk modeling and may affect the mass calculation. Here we derive disk masses assuming the disk-clearing model discussed above and compare them to masses derived assuming continuous disks. Because disk 
clearing has little effect on disk mass estimates for wider binaries, we derive masses only for Sco-Oph binaries with $a_{p}<300$ AU.

The temperature distribution of the disk is determined by fitting the 10-100 $\mu \mathrm{m}$ data for each system with a power law and determining $q$ and $T_{1}$ from the parameters of the fit (BSCG). Some of the binaries in our sample do not have sufficient infrared data available in the literature for this to be possible, and we could not derive masses for these systems. For the remaining systems, we took published values of $q$ and $T_{1}$ from AM for some sources and used infrared data from the literature to derive $q$ and $T_{1}$ for the others. Though emission at 10-100 $\mu \mathrm{m}$ is likely to be dominated by the inner part of the circumstellar disk, we assume that the derived temperature distribution applies to the whole disk.

To calculate the disk mass in the presence of a gap, we assume the emission from the disk is given by Eq. 3, with the disk surface density given by Eq. 5. We calculate masses for three cases: no clearing, circular-orbit clearing $\left(r_{\text {in }}=0.4 a, r_{\text {out }}=1.8 a\right)$, and eccentric-orbit clearing $\left(r_{\text {in }}=0.2 a, r_{\text {out }}=3 a\right)$. For triple or quadruple systems, we choose the projected separation closest to $50 \mathrm{AU}$ since this pair will most affect the derived disk mass. Following BSCG, we take $p=1.5, \theta=0, r_{0}=0.01 \mathrm{AU}$, and $R_{d}=100 \mathrm{AU}$. Following Beckwith \& Sargent (1991), we use the opacity law $\kappa_{\nu}=0.1(\lambda / 250 \mu \mathrm{m})^{-\beta}$, with $\beta=1$. We then numerically integrate Eq. 3 and vary $\Sigma_{0}$ until the calculated flux matches the observed flux. These masses are given in Table 4 .

The table shows that gap clearing does not greatly affect the derived mass for most systems. We further explored this affect using an ensemble of model binary systems with a range of disk properties. For gaps from $0.4 a$ to $1.8 a$, the derived disk mass typically differs by a factor of two or less from the mass calculated assuming a continuous disk; for gaps from $0.2 a$ to $3 a$, the difference is typically a factor of three or less. For some combinations of disk properties and gap locations, the variations are somewhat greater. Gap clearing can either increase or decrease the mass derived from a given flux, depending on whether the area cleared is efficient or inefficient at radiating submillimeter flux compared to the rest of the disk. Thus, gap clearing introduces an additional uncertainty into disk mass calculations for close binary systems.

\subsection{Circumstellar disks in binary environments}

While gap clearing can plausibly explain the reduction of submillimeter flux from binaries with separations of less than 50-100 AU, the upper limits on fluxes from such 
binaries can be equally well explained if the physical conditions (such as surface densities or temperatures) of disks in closer binaries differ from those in wide binaries. Indeed, one straightforward interpretation of the lack of submillimeter emission is the complete absence of disk material.

However, $2 \mu \mathrm{m}$ and $10 \mu \mathrm{m}$ excesses indicate that circumstellar disks are present in at least 50\% of PMS binaries (Mathieu 1994). Similarly, Simon et al. (1995) find the same binary frequency in systems with and without circumstellar disks based on $K-L$ colors, and Simon \& Prato (1995) find the same frequency of $K-N$ color excesses for single stars as for binaries. But observations at these wavelengths only sample material very near stellar surfaces. In addition the high dust opacities at these wavelengths typically do not permit derivation of surface densities.

Longer wavelength IRAS measurements also support a high frequency of circumstellar disks. Mid-infrared (e.g. $60 \mu \mathrm{m}$ ) flux originates in the inner regions of disks (typically $\leq 10$ $\mathrm{AU}$ ); thus for most of the binaries in our sample $60 \mu \mathrm{m}$ flux originates in circumstellar disks. Figure 6 shows IRAS $60 \mu \mathrm{m}$ flux plotted as a function of projected separation for the Tau-Aur and Sco-Oph binaries. IRAS fluxes or upper limits were taken from Weaver \& Jones (1992), Strom et al. (1989), Clark (1991), Hartmann et al. (1991), Wilking, Lada, \& Young (1989), and the IRAS Point Source Catalog. Filled symbols represent detections and open symbols are upper limits. All fluxes are scaled to a distance of 140 pc. Fourteen systems out of 85 do not have IRAS measurements in any of the above references and are not shown here. These systems are approximately equally divided between wide and close binaries.

Figure 6 has two notable features. First, the fraction of binaries detected at $60 \mu \mathrm{m}$ is much higher than at submillimeter wavelengths. If $60 \mu \mathrm{m}$ flux is taken to originate in disks, then at least one circumstellar disk is present in most PMS binaries. As such, the low level of submillimeter emission from the close binaries is not the result of a total absence of disk material. Second, there is no marked dependence of $60 \mu \mathrm{m}$ flux on binary separation akin to that seen at submillimeter wavelengths. The same two-sample tests performed on the submillimeter data show no difference in the $60 \mu \mathrm{m}$ flux distributions of the close and wide binaries divided at 10, 50, or $100 \mathrm{AU}$.

These $60 \mu \mathrm{m}$ flux measurements can provide meaningful constraints on the surfacedensity normalizations of circumstellar disks. Because dust opacity is much higher at 60 $\mu \mathrm{m}$ than at submillimeter wavelengths, disks remain optically thick at $60 \mu \mathrm{m}$ to much lower surface densities than in the submillimeter. An explicit assumption of previous analyses of disk masses is that the disks are optically thick at $60 \mu \mathrm{m}$ and thus reflect the disk temperature distributions. However, given only an upper limit on submillimeter flux and 
thus circumstellar disk surface density, this assumption need not hold. For a power-law radial temperature distribution one signature of optically thin $60 \mu \mathrm{m}$ emission would be a steepening of the spectral slope between $12 \mu \mathrm{m}$ and $60 \mu \mathrm{m}$. In fact, several binaries show such steepening at a formally significant level given the quoted uncertainties on the IRAS fluxes. Thus some circumstellar disks may be partially optically thin at $60 \mu \mathrm{m}$. However, given that other binaries also show deviations from single spectral slopes in other senses (e.g., high $12 \mu \mathrm{m}, 25 \mu \mathrm{m}$, or $60 \mu \mathrm{m}$ fluxes at formally significant levels), we do not feel that in any given case the conclusion of optical thinness is secure.

Rather we choose to use the binaries with steepening spectral slopes to derive lower limits on masses and surface densities of circumstellar disks. Specifically we have chosen three Tau-Aur binaries with $a_{p}<50 \mathrm{AU}$ and for which the slope of $\lambda F_{\lambda}$ from 25 to $60 \mu \mathrm{m}$ is steeper than the slope from 12 to $25 \mu \mathrm{m}$ : DF Tau, FO Tau, and CZ Tau. These binaries have the highest quality IRAS flux measurements and no additional companions. The IRAS fluxes of these three binaries lie in the middle of the range of detected fluxes, and thus we take these systems to be representative examples of young binary systems. For each binary we have derived disk masses using the method described in $\$ 5.1 .2$., except that the disk temperature was derived from only the IRAS 12 and $25 \mu \mathrm{m}$ fluxes and the disk radius was taken to be half the projected binary separation. The disk surface density was then varied so that the model flux matched the observed $60 \mu \mathrm{m}$ flux. We used the dust opacity law given by Adams et al. (1988) since Beckwith \& Sargent (1991) do not give opacities for IRAS wavelengths.

The circumstellar disk masses derived for these binaries range from $5 \times 10^{-6} M_{\odot}$ to $7 \times 10^{-5} M_{\odot}$. These numbers cannot be directly compared with masses derived for disks around single stars because of the smaller disk radii used here. A more significant comparison is the disk surface-density normalization $\left(\Sigma_{0}\right.$; Eq. 5). The derived surfacedensity normalizations for these circumstellar disks are roughly two orders of magnitude smaller than those for disks around single stars detected at submillimeter wavelengths. The essential conclusion to be drawn from this analysis is that the mechanism reducing the submillimeter flux from binaries with $1<a_{p}<50-100$ AU does not entirely destroy circumstellar disks or inhibit their formation.

We stress that these mass and surface density estimates are best considered as lower limits. While the steepening spectral slopes of these binaries may be indicative of partial optical thinness at $60 \mu \mathrm{m}$ as presumed in these calculations, we cannot rule out fluctuations

${ }^{7}$ CZ Tau lies 30 " from DD Tau, but Weaver \& Jones (1992) list CZ Tau as a "better positional fit" to the IRAS source. 
in the IRAS fluxes larger than the formal errors. At the same time, we have shown that in the presence of gaps the submillimeter flux upper limits do not require that the circumstellar surface densities be significantly lower than found in disks around single stars. Thus, the available data require circumstellar disk surface densities in close binaries to be in a range from $1 \%$ to the same as typical disks around single stars. These limits constrain the degree of depletion of circumstellar disks by the various processes discussed in the Introduction.

The lack of dependence of $60 \mu \mathrm{m}$ emission on binary separation is not inconsistent with the gap model. The specific model shown in Figure 5 d predicts a factor of $\sim 5$ decrease in $60 \mu \mathrm{m}$ flux for binaries with separations between $1 \mathrm{AU}$ and $10 \mathrm{AU}$. Given the small number of systems in this separation range, such a change would be undetectable with current data. In addition, as noted previously most binaries at these small separations were discovered via lunar occultation, so that their true separations may be substantially underestimated.

Finally, it is plausible that the temperature distributions of disks in close binary environments would differ from those in wide binaries or around single stars, also influencing submillimeter and infrared emission. We used the $T_{1}$ and $q$ values (see Eq. 1) for the Sco-Oph and Tau-Aur binaries to investigate whether the temperature parameters vary systematically with separation. We find no evidence for a dependence of disk temperature on binary separation. These parameters only provide information about the inner 10 AU or so of the disk. The temperature distribution of the disks elsewhere - and in particular at radii comparable to most binary separations - is largely unconstrained by current observations.

\subsection{Circumbinary disks}

Because of their low temperatures, circumbinary disks in all but the closest binaries emit predominantly at far-infrared and longer wavelengths. However, since substantial emission at these wavelengths can arise from a large range of radii within a disk, securely associating unresolved flux measurements with circumbinary disks can be problematic. On the other hand, upper limits on submillimeter emission unambiguously place upper limits on circumbinary disk masses. For optically-thin emission at $\lambda=1300 \mu \mathrm{m}$ with $\kappa_{\nu}=0.02$ $\mathrm{cm}^{2} \mathrm{~g}^{-1}$, the circumbinary mass $M_{c b}$ that produces a given flux $F_{\nu}$ is

$$
M_{c b}=2.6 \times 10^{-4} M_{\odot}\left(F_{\nu} / \mathrm{mJy}\right)\left(e^{(11.1 \mathrm{~K} / T)}-1\right)
$$

assuming a distance of $140 \mathrm{pc}$ and emission in the Rayleigh limit. Taking $T=15 \mathrm{~K}$ as a minimum dust temperature and $F_{\nu}<15$ mJy as a typical flux upper limit for binaries 
with separations of less than $\sim 100 \mathrm{AU}$, we find $M_{c b}<4.3 \times 10^{-3} M_{\odot}$. This upper limit is conservative given that some emission may derive from a circumstellar disk. On the other hand if the circumbinary material were optically thick, the derived mass limit would be higher. Thus we conclude that binaries with projected separations between a few AU and $\sim 100$ AU typically do not have circumbinary disks with masses greater than $0.005 \mathrm{M}_{\odot}$.

There is one notable counterexample, however. As discussed above, a ring-like circumbinary disk around the 40 AU binary GG Tau has been resolved at $3 \mathrm{~mm}$ (Dutrey et

al. 1994). The mass derived for this ring from the continuum emission is $0.13 M_{\odot}$. GG Tau is unusual in being the most luminous submillimeter source in our sample (Figure 3) and one of the younger binaries in Tau-Aur. Most PMS binaries of similar projected separation do not have similarly massive or luminous circumbinary disks, suggesting the possibility that GG Tau represents a brief phase in early circumbinary disk evolution.

Circumbinary disks have also been found around several very close binaries. For example, in HP Tau (one of the closest binaries in our sample with detected $1300 \mu \mathrm{m}$ emission), a simple calculation shows that a circumbinary disk must be present. If the binary orbit were filled with optically-thick material emitting at the stellar temperature, the resulting submillimeter flux would be comparable to that observed; however, the optical flux of such a quantity of hot material would be $\sim 10^{4}$ times the observed flux. Thus, the submillimeter-emitting region in HP Tau must be substantially larger than a few AU in radius and therefore must lie primarily outside the binary orbit. A similar calculation requires the presence of circumbinary disks around the PMS spectroscopic binaries GW Ori (Mathieu et al. 1995), AK Sco and V4046 Sgr (detected in this work), and DQ Tau (Mathieu 1995).

To conclude, our flux limits place upper limits on circumbinary disk masses of 0.005 $M_{\odot}$ for most binaries with separations of less than roughly $100 \mathrm{AU}$. Indeed, it remains possible that most binaries do not have circumbinary disks. The present submillimeter flux detections and upper limits for almost all binaries with separations greater than a few AU are entirely consistent with emission from only circumstellar disks.

\section{Conclusion}

We have made sensitive $800 \mu \mathrm{m}$ continuum observations of most (25) pre-main-sequence binaries with projected separations $a_{p} \lesssim 150 \mathrm{AU}$ in the Scorpius-Ophiuchus star-forming region, and we have supplemented these data with previous $1300 \mu \mathrm{m}$ continuum observations 
to obtain a sample of 30 systems. We have also created a similar database from the literature for 55 systems in Taurus-Auriga. We have used these data to study the nature of disks in young binary environments, and we find:

1) Submillimeter fluxes from binaries with $1<a_{p}<50-100 \mathrm{AU}$ are lower on average than from wider binaries or single stars, whereas the flux distributions of wide binaries and single stars are indistinguishable. This dependence of submillimeter flux on binary separation is seen independently in the Sco-Oph and Tau-Aur samples. When the samples are combined the effect is found at greater than the $99 \%$ confidence level. The transition separation of 50-100 AU is similar to typically derived radii for dust disks around young stars, strongly suggesting that the reduction in submillimeter emission among closer binaries is due to the influence of the companions on disks.

2) The reduction in submillimeter flux from binaries with $1<a_{p}<50-100$ AU can plausibly be attributed to gaps cleared in disks by binaries with eccentric orbits. As such, the present upper limits permit but do not require a large reduction in the surface densities of disk material outside such gaps compared to surface densities of disks among wide binaries or single stars.

3) Most of the binaries in our sample were detected at $60 \mu \mathrm{m}$ by IRAS, indicating that each of these binaries has at least one circumstellar disk. Presuming the disks to be optically thick at $60 \mu \mathrm{m}$, the flux measurements place lower limits of roughly $10^{-5} M_{\odot}$ on circumstellar disk masses. This lower limit corresponds to circumstellar disk surface densities no more than two orders of magnitude smaller than surface densities of most disks detected at submillimeter wavelengths.

4) We place upper limits of $0.005 M_{\odot}$ on circumbinary disk masses around most binaries with projected separations between 1 AU and 100 AU. Circumbinary disks as massive as that found around GG Tau are rare for these binaries. However, submillimeter detections of binaries with separations less than a few AU show that massive circumbinary disks can exist around the closest binaries.

The present body of data is consistent with the following picture for disks in PMS binary environments. Binaries with semimajor axes of a few hundred AU or greater have circumstellar disks with properties very similar to those of disks found around single stars. Binaries with semimajor axes between a few AU and 50-100 AU also typically have at least one circumstellar disk each. The binaries truncate these disks, thus limiting their submillimeter emission. Their surface densities remain uncertain within a range of 0.01 to 1 times the surface densities of disks around single stars. Binaries in this separation range typically do not have massive $\left(M>0.005 M_{\odot}\right)$ circumbinary disks. Binaries with 
semimajor axes of less than a few AU can have massive circumbinary disks. Such binaries truncate the inner edges of circumbinary disks at radii of only a few AU or less, leaving most of the disk undisturbed on dynamical timescales.

Notably absent from this morphological picture is any discussion of the detailed physical conditions of the disks, such as temperature, surface-density, and opacity distributions in both circumstellar and circumbinary disks. In addition to clearing gaps, companions are expected to influence these disk properties (e.g. Syer \& Clarke 1995). At the same time, the present submillimeter flux upper limits can only just be explained by our model, even assuming relatively large gaps driven by eccentric binaries. We anticipate that disks in young binary environments are substantially more complex than the simple disk model employed here.

Finally, we note that binaries are the primary product of star formation, and thus the frequency of other planetary systems is intimately linked to the issues raised here. For binaries with separations of less than 100 AU, present upper limits on submillimeter flux imply upper limits on total disk mass of a few times $10^{-3} M_{\odot}$. This is roughly an order of magnitude smaller than typical estimates for the minimum mass of the early solar nebula (e.g. Weidenschilling 1977). Such a disk could conceivably form terrestrial planets, but it is unlikely to form gaseous giant planets. However, these mass estimates are insensitive to material in grains or planetesimals larger than a few $\mathrm{mm}$ in size. Thus, it remains possible that close binaries can form planets, but it would appear that sufficient disk material to form planetary systems like our own is most likely to be found in wide binaries or single stars.

We thank the referee, Steve Beckwith, for useful comments which improved the presentation of this paper. We are grateful to the staff of the JCMT for their knowledgeable support and good humor. ELNJ gratefully acknowledges the support of a Grant-in-Aid of Research from the National Academy of Sciences through Sigma Xi, as well as funding from the National Space Grant College and Fellowship Program and the Wisconsin Space Grant

Consortium. RDM appreciates funding from the Presidential Young Investigator program, a Guggenheim Fellowship, the Morrison Fund of Lick Observatory, and the Wisconsin Alumni Research Fund. GAF acknowledges the support of an NRAO Jansky Fellowship. This research has made use of the Simbad database, operated at CDS, Strasbourg, France.

\section{A. Appendix: Observations of pre-main-sequence stars in other regions}


During the course of the JCMT observations reported above, we surveyed a number of other PMS binaries at submillimeter wavelengths. These systems were excluded from the main sample discussed above because they are located in star-forming regions other than Sco-Oph or Tau-Aur, or because they have spectral types earlier than F0. Submillimeter fluxes for these systems are given in Table 5 and each is discussed briefly below. While we did not make a systematic survey, our detections of $800 \mu \mathrm{m}$ emission from the isolated $\mathrm{T}$ Tauri stars Hen 3-600, HD 98800, and V4046 Sgr are interesting. If these systems were formed from small, isolated molecular clouds, our detections suggest that disk survival times (at least in binary systems) may be longer than the cloud dispersal times.

V4046 Sgr: V4046 Sgr (HDE 319139, HBC 662) was discovered to be a spectroscopic binary by Byrne (1986) and de la Reza et al. (1986). It is the shortest-period PMS binary detected at submillimeter wavelengths to date and is also one of the brightest PMS binary sources at these wavelengths. The strength of the submillimeter emission requires the presence of a circumbinary disk (see \$5.3.). V4046 Sgr is not associated with any known region of star formation; it lies $1^{\circ}$ from the nearest (small) dark cloud and is not near any CO clouds (de la Reza et al. 1986). However, it does show characteristics typical of classical T Tauri stars: strong, variable $\mathrm{H} \alpha$ emission, Li absorption, and UV and infrared excesses (de la Reza et al. 1986). V4046 Sgr has a disk mass comparable to those found around other $\mathrm{T}$ Tauri stars, another piece of evidence that it is indeed a pre-main-sequence binary.

CoD $-\mathbf{2 9}^{\circ} \mathbf{8 8 8 7}$, CoD $-\mathbf{3 3}^{\circ} \mathbf{7 7 9 5}$, Hen $3-600$ : These stars are isolated $\mathrm{T}$ Tauri stars located in the vicinity of TW Hya (de la Reza et al. 1989, Gregorio-Hetem et al. 1992). All have $\mathrm{H} \alpha$ emission and $\mathrm{Li}$ absorption, but they are not located near any known star-forming region and are at moderately high $\left(21^{\circ}-28^{\circ}\right)$ galactic latitude. Hen 3-600, the only one of the three detected at $800 \mu \mathrm{m}$, is also the only one detected by IRAS. Both CoD $-29^{\circ} 8887$ and Hen 3-600 are binaries (de la Reza et al. 1989, Reipurth \& Zinnecker 1993).

HD 98800: This quadruple system (Torres et al. 1995) is another isolated pre-mainsequence system in the vicinity of TW Hya. Zuckerman \& Becklin (1993) noted that its infrared excess is large for a Vega-excess system; however, the excess is not unusual for a PMS system. Fekel \& Bopp (1993) classify it as pre-main-sequence with an estimated age of less than $10^{7} \mathrm{yr}$. The $800 \mu \mathrm{m}$ flux measured by us is consistent with that reported by Rucinski (1993). Our $800 \mu \mathrm{m}$ flux measurement, combined with the $1100 \mu \mathrm{m}$ measurement by Stern, Weintraub, \& Festou (1994), yields disk masses of $\sim 10^{-3}-10^{-4} M_{\odot}$ for assumed distances of $20-60 \mathrm{pc}$.

BZ Sgr: This $5^{\prime \prime}$ binary was discovered to be a high-latitude $\mathrm{T}$ Tauri system by 
Gregorio-Hetem et al. (1992). Our detection of $800 \mu \mathrm{m}$ emission is additional evidence that it is a PMS system.

FK Ser: A 1".33 PMS binary (Herbig \& Bell 1988), this system was classified as "post-T Tauri" by Herbig (1973) but has since been considered a normal T Tauri star in many references (e.g. Herbig \& Bell 1988). Our detection of $800 \mu \mathrm{m}$ emission further suggests that FK Ser has disk properties similar to those of other T Tauri stars.

S CrA: This 1".4 PMS binary has a spectral type of K6 and lies in the Corona Australis star-forming region (Herbig \& Bell 1988).

HD 150193 (MWC 863, Elias 2-49): This Herbig Ae/Be binary has a spectral type of A0 (Elias 1978) and a projected separation of 1".1 (Reipurth \& Zinnecker 1993).

CoKu Ser/G1: This 3.5 PMS binary has a spectral type of K7 and lies in the Serpens molecular cloud (Herbig \& Bell 1988). 
Table 1: JCMT FLuXes or $3 \sigma$ UPPER LIMITS FOR SCO-Oph BINARIES

\begin{tabular}{|c|c|c|c|c|c|}
\hline Name & $\begin{array}{l}\text { Projected } \\
\text { Sep. }(\mathrm{AU})^{\mathrm{a}}\end{array}$ & $\begin{array}{c}F_{\nu}(1100 \mu \mathrm{m}) \\
(\mathrm{mJy})\end{array}$ & $\begin{array}{c}F_{\nu}(800 \mu \mathrm{m}) \\
(\mathrm{mJy})\end{array}$ & $\begin{array}{c}F_{\nu}(450 \mu \mathrm{m}) \\
(\mathrm{mJy})\end{array}$ & $\begin{array}{c}F_{\nu}(350 \mu \mathrm{m}) \\
(\mathrm{mJy})\end{array}$ \\
\hline $155203-2338$ & 128 & $\cdots$ & $<30$ & $\cdots$ & $\cdots$ \\
\hline $155808-2219$ & 0.048 & $\ldots$ & $<26$ & $\ldots$ & $\ldots$ \\
\hline $155913-2233^{\mathrm{b}}$ & $0.014 / 46.1$ & $\ldots$ & $<26$ & $\ldots$ & $\ldots$ \\
\hline $160814-1857$ & 0.19 & $\cdots$ & $<36$ & $\cdots$ & $\cdots$ \\
\hline $160905-1859$ & 0.015 & $\cdots$ & $<34$ & $\cdots$ & $\cdots$ \\
\hline $160946-1851$ & 33.3 & $\ldots$ & $<38$ & $\ldots$ & $\ldots$ \\
\hline $162218-2420$ & 29.5 & $\ldots$ & $<39$ & $\ldots$ & $\ldots$ \\
\hline $162814-2427^{\mathrm{b}}$ & $0.27 / 19.4$ & $\ldots$ & $<16$ & $\ldots$ & $\ldots$ \\
\hline $162819-2423^{\mathrm{b}}$ & $0.10 / 2 / 600$ & $<30$ & $<17$ & $\ldots$ & $\ldots$ \\
\hline AK Sco & 0.143 & $36 \pm 4$ & $83 \pm 9$ & $242 \pm 32$ & $\ldots$ \\
\hline AS 205 & 211 & $503 \pm 17$ & $998 \pm 43$ & $2541 \pm 190$ & $3515 \pm 446$ \\
\hline DoAr 24E & 257 & $\ldots$ & $37 \pm 8$ & $\ldots$ & $\ldots$ \\
\hline Elias 2-26 & 150 & $\ldots$ & $<58$ & $\ldots$ & $\ldots$ \\
\hline Haro 1-4 & 90.0 & $\cdots$ & $53 \pm 9$ & $<361$ & $\ldots$ \\
\hline ROX 31 & 60.0 & $<76$ & $<22$ & $\ldots$ & $\ldots$ \\
\hline SR 9 & 73.8 & $\ldots$ & $<28$ & $\cdots$ & $\cdots$ \\
\hline SR 12 & 37.5 & $<62$ & $<26$ & $\cdots$ & $\cdots$ \\
\hline SR 20 & 8.9 & $\ldots$ & $<28$ & $\ldots$ & $\cdots$ \\
\hline V853 Oph & $1.6 / 53.8$ & $52 \pm 8$ & $134 \pm 12$ & $320 \pm 53$ & $\cdots$ \\
\hline VV Sco & 240 & $\ldots$ & $<63$ & $\ldots$ & $\ldots$ \\
\hline WSB 3 & 75.0 & $\cdots$ & $<34$ & $\cdots$ & $\cdots$ \\
\hline WSB 11 & 62.5 & $\cdots$ & $<26$ & $\ldots$ & $\ldots$ \\
\hline WSB 18 & 138 & $\ldots$ & $<46$ & $\ldots$ & $\ldots$ \\
\hline WSB 19 & 188 & $\ldots$ & $<78$ & $\ldots$ & $\ldots$ \\
\hline WSB 26 & 150 & $\cdots$ & $<77$ & $\cdots$ & $\cdots$ \\
\hline
\end{tabular}

${ }^{a}$ Projected separations greater than $1 \mathrm{AU}$ are based on measured angular separations and assume distances of $125 \mathrm{pc}$ to $\rho$ Oph and $160 \mathrm{pc}$ to Upper Sco. Projected separations less than 1 AU are from spectroscopic orbits and are projected semimajor axes $a \sin i$.

${ }^{b}$ These multiple systems have pairs with projected separations both less than and greater than the $1 \mathrm{AU}$ sample cutoff. For the purposes of the statistical analysis, the pairs with $a_{p}<1$ AU are ignored. 
Table 2: SurveYs FOR BINARIES IN SCO-OpH

\begin{tabular}{lcll} 
Survey & Separation Range & Technique & Target List \\
\hline Mathieu et al. (1989) & $0.01-\sim 2 \mathrm{AU}$ & $\begin{array}{l}\text { Optical } \\
\text { spectroscopy }\end{array}$ & $\begin{array}{l}\text { X-ray selected stars, primarily } \\
\text { naked T Tauri stars }\end{array}$ \\
Ghez et al. (1993) & $14-250 \mathrm{AU}$ & $\begin{array}{l}2.2 \mu \mathrm{m} \text { speckle } \\
\text { interferometry }\end{array}$ & $\begin{array}{l}\text { HBC and x-ray selected stars } \\
\text { from Walter et al. (1994) }\end{array}$ \\
$\begin{array}{l}\text { Reipurth \& } \\
\text { Zinnecker (1993) }\end{array}$ & $140-1700 \mathrm{AU}$ & $0.9 \mu \mathrm{m}$ imaging & $\begin{array}{l}\text { H } \alpha \text { emission stars from } \\
\text { Wilking, Schwartz, \& Blackwell } \\
(1987) \text { and Walter }(1986)\end{array}$ \\
Simon et al. (1992, 1995) & $0.7-1400 \mathrm{AU}$ & $\begin{array}{l}\text { Lunar occultation, } \\
2.2 \mu \mathrm{m} \text { imaging }\end{array}$ & $\begin{array}{l}\text { Members of } \rho \text { Oph from IR } \\
\mathrm{H} \alpha \text { and x-ray surveys }\end{array}$
\end{tabular}

${ }^{a}$ Assuming a distance of $140 \mathrm{pc}$. 
Table 3: Confidence Levels From two-SAMPle tests

\begin{tabular}{llrr}
\hline \hline Groups & Region & $\begin{array}{r}\text { Number } \\
\text { in groups }\end{array}$ & $\begin{array}{r}\text { Confidence level } \\
\text { that groups differ }\end{array}$ \\
\hline \multirow{2}{*}{ Binaries with $a_{p} \leq 50$ AU vs. $a_{p}>50$ AU } & Tau-Aur & $28 / 33$ & $97-98 \%$ \\
& Sco-Oph & $10 / 22$ & $89-95 \%$ \\
Binaries with $a_{p} \leq 100$ AU vs. $a_{p}>100$ AU & Combined & $38 / 55$ & $99-99.8 \%$ \\
& Tau-Aur & $37 / 24$ & $98-99 \%$ \\
& Sco-Oph & $17 / 15$ & $91-95 \%$ \\
Binaries with $a_{p} \leq 50$ AU vs. single stars & Combined & $54 / 39$ & $99.6-99.9 \%$ \\
& Tau-Aur & $28 / 55$ & $99.5-99.7 \%$ \\
& Sco-Oph & $10 / 47$ & $93-98 \%$ \\
Binaries with $a_{p} \leq 100$ AU vs. single stars & Combined & $38 / 102$ & $99.9-99.96 \%$ \\
& Tau-Aur & $37 / 55$ & $99.5-99.6 \%$ \\
& Sco-Oph & $17 / 47$ & $93-97 \%$ \\
Binaries with $a_{p}>50$ AU vs. single stars & Combined & $54 / 102$ & $99.92-99.97 \%$ \\
& Tau-Aur & $33 / 55$ & $54-74 \%$ \\
& Sco-Oph & $22 / 47$ & $31-51 \%$ \\
Binaries with $a_{p}>100$ AU vs. single stars & Combined & $55 / 102$ & $51-75 \%$ \\
& Tau-Aur & $24 / 55$ & $22-54 \%$ \\
& Sco-Oph & $15 / 47$ & $3-10 \%$ \\
& Combined & $39 / 102$ & $<1-33 \%$ \\
\hline
\end{tabular}


Table 4: Disk MASSES FROM VARIOUS GAP CLEARING MODELS

\begin{tabular}{|c|c|c|c|c|c|}
\hline \multirow[t]{2}{*}{ Name } & \multirow{2}{*}{$\begin{array}{c}\text { Proj. Sep. } \\
(\mathrm{AU})\end{array}$} & \multirow{2}{*}{$\begin{array}{c}\lambda \\
(\mu \mathrm{m})\end{array}$} & \multicolumn{3}{|c|}{ Disk Mass $\left(10^{-3} M_{\odot}\right)$} \\
\hline & & & No clearing & Gap $0.4 a-1.8 a$ & Gap $0.2 a-3 a$ \\
\hline $162819-2423$ & 2.0 & 800 & $<0.5$ & $<0.8$ & $<1.2$ \\
\hline SR 20 & 8.9 & 800 & $<2.1$ & $<3.9$ & $<7.5$ \\
\hline $162814-2427$ & 19.4 & 800 & $<0.4$ & $<0.4$ & $<0.4$ \\
\hline $\mathrm{SR} 24 \mathrm{~N}$ & 24.6 & 1300 & $<1.9$ & $<2.2$ & $<4.1$ \\
\hline $160946-1851$ & 33.3 & 800 & $<2.8$ & $<3.2$ & \\
\hline SR 12 & 37.5 & 800 & $<0.3$ & $<0.3$ & $<0.2$ \\
\hline V853 Oph & 53.8 & 800 & 5.2 & 5.3 & $\cdots$ \\
\hline SR 9 & 73.8 & 1300 & 1.6 & 1.2 & 1.1 \\
\hline Haro 1-4 & 90.0 & 800 & 1.5 & 1.1 & 1.1 \\
\hline AS 205 & 211 & 800 & 54.3 & 56.6 & $\cdots$ \\
\hline VV Sco & 240 & 800 & $<7.7$ & $<7.5$ & $<6.4$ \\
\hline DoAr $24 \mathrm{E}$ & 257 & 800 & 0.5 & 0.5 & 0.4 \\
\hline
\end{tabular}

${ }^{a}$ The flux from an optically-thick disk with this clearing is less than the measured flux or flux limit, so no mass could be calculated.

Table 5: JCMT FLUXES FOR OTHER PRE-MAIN-SEQUENCE STARS

\begin{tabular}{lccccc}
\hline Name & $\begin{array}{c}\text { Projected } \\
\text { Sep. }(\text { arcsec })\end{array}$ & $\begin{array}{c}F_{\nu}(1100 \mu \mathrm{m}) \\
(\mathrm{mJy})\end{array}$ & $\begin{array}{c}F_{\nu}(800 \mu \mathrm{m}) \\
(\mathrm{mJy})\end{array}$ & $\begin{array}{c}F_{\nu}(450 \mu \mathrm{m}) \\
(\mathrm{mJy})\end{array}$ & $\begin{array}{c}F_{\nu}(350 \mu \mathrm{m}) \\
(\mathrm{mJy})\end{array}$ \\
\hline \hline BZ Sgr & 5 & $\ldots$ & $116 \pm 26$ & $\ldots$ & $\ldots$ \\
CoD -29॰8887 & $\leq 0.8$ & $\ldots$ & $<60$ & $\ldots$ & $\ldots$ \\
CoD -337795 & single & $\ldots$ & $<126$ & $\ldots$ & $\ldots$ \\
CoKu Ser/G1 & 3.5 & $\ldots$ & $<147$ & $\ldots$ & $\ldots$ \\
FK Ser & 1.33 & $<47$ & $51 \pm 8$ & $<818$ & $\ldots$ \\
HD 150193 & 1.1 & $\ldots$ & $123 \pm 18$ & $455 \pm 150$ & $\ldots$ \\
HD 98800 & $1.5 /$ SB1/SB2 & $\ldots$ & $108 \pm 13$ & $<365$ & $\ldots$ \\
Hen 3-600 & 1.4 & $\ldots$ & $53 \pm 15$ & $\ldots$ & $\ldots$ \\
S CrA & 1.4 & $361 \pm 26$ & $765 \pm 51$ & $2750 \pm 383$ & $\ldots$ \\
V4046 Sgr & SB2 & $451 \pm 20$ & $770 \pm 39$ & $2042 \pm 111$ & $3154 \pm 419$ \\
\hline
\end{tabular}




\section{REFERENCES}

Abt, H. A. 1983, ARA\&A, 21, 343

Adams, F. C., Emerson, J. P., \& Fuller, G. A. 1990, ApJ, 357, 606

Adams, F. C., Lada, C. J., \& Shu, F. H. 1988, ApJ, 326, 865

Agladze, N. I., Sievers, A. J., Jones, S. A., Burlitch, J. M., \& Beckwith, S. V. W. 1994, Nature, 372, 243

Andersen, J., Lindgren, H., Hazen, M. L., \& Mayor, M. 1989, A\&A, 219, 142

André, P., \& Montmerle, T. 1994, ApJ, 420, 837 (AM)

André, P., Montmerle, T., Feigelson, E. D., \& Steppe, H. 1990, A\&A, 240, 321

Artymowicz, P., Clarke, C. J., Lubow, S. H., \& Pringle, J. E. 1991, ApJ, 370, L35

Artymowicz, P., \& Lubow, S. H. 1994, ApJ, 421, 651

Artymowicz, P., \& Lubow, S. H. 1995, in Disks and Outflows Around Young Stars, ed. Beckwith, S. V. W., Natta, A., \& Staude, H. J. (Berlin: Springer-Verlag) in press

Barsony, M., Burton, M. G., Russell, A. P. G., Carlstrom, J. E., \& Garden, R. 1989, ApJ, 346, L93

Basri, G., \& Bertout, C. 1993, in Protostars and Planets III, ed. E. H. Levy \& J. I. Lunine (Tucson: The University of Arizona Press), 543

Beckwith, S. V. W., \& Sargent, A. I. 1991, ApJ, 381, 250

Beckwith, S. V. W., \& Sargent, A. I. 1993, in Protostars and Planets III, ed. E. H. Levy \& J. I. Lunine (Tucson: The University of Arizona Press), 521

Beckwith, S., Sargent, A., Chini, R., \& Güsten, R. 1990, AJ, 99, 924 (BSCG)

Blauuw, A. 1978, in Problems of Physics and Evolution of the Universe, ed. L. V. Mirzoyan (Yerevan: Armenian Academy of Sciences), 101

Byrne, P. B. 1986, Irish Astron. J., 17, 294

Clark, F. O. 1991, ApJS, 75, 611

Clarke, C. J. 1992, in Complementary Approaches to Double and Multiple Star Research, IAU Colloquium 135, ed. H. A. McAlister \& W. I. Hartkopf (San Francisco: Astronomical Society of the Pacific), 176

de Geus, E. J., de Zeeuw, P. T., \& Lub, J. 1989, A\&A, 216, 44

de la Reza, R., Quast, G., Torres, C. A. O., Mayor, M., Meylan, G., \& Llorente de Andrés, F. 1986, New Insights in Astrophysics (Noordwijk: ESA), 107 
de la Reza, R., Torres, C. A. O., Quast, G., Castilho, B. V., \& Vieira, G. L. 1989, ApJ, 343, L61

Duquennoy, A., \& Mayor, M. 1991, A\&A, 248, 485

Dutrey, A., Guilloteau, S., \& Simon, M. 1994, A\&A, 286, 149

Elias, J. H. 1978, ApJ, 224, 453

Feigelson, E. D., \& Nelson, P. I. 1985, ApJ, 293, 192

Fekel, F. C., \& Bopp, B. W. 1993, ApJ, 419, L89

Ghez, A. M., Neugebauer, G., \& Matthews, K. 1993, AJ, 106, 2005

Gregorio-Hetem, J., Lépine, J. R. D., Quast, G. R., Torres, C. A. O., \& de la Reza, R. 1992, AJ, 103, 549

Hartmann, L. W., Jones, B. F., Stauffer, J. R., \& Kenyon, S. J. 1991, AJ, 101, 1050

Herbig, G. H. 1973, ApJ, 182, 129

Herbig, G. H., \& Bell, K. R. 1988, Lick Observatory Bulletin 1111

Jensen, E. L. N., Koerner, D. W., \& Mathieu, R. D. 1995, in preparation

Jensen, E. L. N., Mathieu, R. D., \& Fuller, G. F. 1994, ApJ, 429, L29

Koresko, C. D. 1995, ApJ, 440, 764

Lada, C. J. 1987, in IAU Symposium 115, Star Forming Regions, ed. M. Peimbert \& J. Jugaku (Dordrecht: Kluwer), 1

LaValley, M., Isobe, T., \& Feigelson, E. D. 1992, BAAS, 24, 839

Leinert, Ch., Weitzel, N., Zinnecker, H., Christou, J., Ridgeway, S., Jameson, R., Haas, M., \& Lenzen, R. 1993, A\&A, 278, 129

Lin, D. N. C., \& Papaloizou, J. 1993, in Protostars and Planets III, ed. E. H. Levy \& J. I. Lunine (Tucson:University of Arizona Press), 749

Mathieu, R. D. 1994, ARA\&A, 32, 465

Mathieu, R. D. 1995, private communication

Mathieu, R. D., Adams, F. C., Fuller, G.A., Jensen, E. L. N., Koerner, D. W., \& Sargent, A. I. 1995, AJ, 109, 2655

Mathieu, R. D., Walter, F. M., \& Myers, P. C. 1989, AJ, 98, 987

Osterloh, M., \& Beckwith, S. V. W. 1995, ApJ, 439, 288

Ostriker, E. C., Shu, F. H., \& Adams, F. C. 1992, ApJ, 399, 192

Phillips, R. B., Lonsdale, C. J., \& Feigelson, E. D. 1991, ApJ, 382, 261 
Pollack, J. B., Hollenbach, D., Beckwith, S., Roush, T., \& Fong, W. 1994, ApJ, 421, 615

Pringle, J. 1991, MNRAS, 248, 754

Reipurth, B., \& Zinnecker, H. 1993, A\&A, 278, 81

Reipurth, B., Chini, R., Krügel, E., Kreysa, E., \& Sievers, A. 1993, A\&A, 273, 221

Rieke, G. H., Ashok, N. M., \& Boyle, R. P. 1989, ApJ, 339, L71

Rucinski, S. M. 1993, IAU Circ. 5788

Sargent, A. I. 1995, in Disks and Outflows Around Young Stars, ed. Beckwith, S. V. W., Natta, A., \& Staude, H. J. (Berlin: Springer-Verlag) in press

Simon, M., Chen, W. P., Howell, R. R., \& Slovik, D. 1992, ApJ, 384, 212

Simon, M., Ghez, A. M., Leinert, Ch., Cassar, L., Chen, W. P., Howell, R. R., Jameson, R. F., Matthews, K., Neugebauer, G., \& Richichi, A. 1995, ApJ, 443, 625

Simon, M., \& Prato, L. 1995, ApJ, in press

Stern, S. A., Weintraub, D. A., \& Festou, M. C. 1994, IAU Circ. 6003

Strom, K. M., Strom, S. E., Edwards, S., Cabrit, S., \& Skrutskie, M. F. 1989, AJ, 97, 1451

Syer, D., and Clarke, C. J. 1995, MNRAS, in press

Terebey, S., Chandler, C. J., \& André, P. 1993, ApJ, 414, 1

Torres, G., Stefanik, R. P., Latham, D. W., \& Mazeh, T. 1995, preprint

Walter, F. M. 1986, ApJ, 306, 573

Walter, F. M., Vrba, F. J., Mathieu, R. D., Brown, A., \& Myers, P. C. 1994, AJ, 107, 692

Weaver, W. B., \& Jones, G. 1992, ApJS, 78, 239

Weidenschilling, S. J. 1977, Ap\&SS, 51, 153

Wilking, B. A., Lada, C. J., \& Young, E. T. 1989, ApJ, 340, 823

Wilking, B. A., Schwartz, R. D., \& Blackwell, J. H. 1987, AJ, 94, 106

Zuckerman, B., \& Becklin, E. E. 1993, ApJ, 406, L25 
Fig. 1. - Submillimeter flux vs. binary separation for Scorpius-Ophiuchus pre-main-sequence binaries. Filled symbols are detections, open symbols are $3 \sigma$ upper limits. Squares are $1300-\mu \mathrm{m}$ fluxes and triangles are $800 \mu \mathrm{m}$ fluxes scaled to $1300 \mu \mathrm{m}$ assuming optically-thick emission in the Rayleigh limit. All fluxes are scaled to a distance of 140 pc. A "T" next to a point indicates that it represents the projected separation of one pair in a triple system. Note the higher fluxes among binaries with $a_{p}>50-100$ AU.

Fig. 2.- Submillimeter flux vs. binary separation for Taurus-Auriga pre-main-sequence binaries. Symbols are as in Figure 1.

Fig. 3.- Submillimeter flux vs. binary separation for the combined sample of Taurus-Auriga and Scorpius-Ophiuchus pre-main-sequence binaries. Symbols are as in Figure 1, with all fluxes scaled to a distance of $140 \mathrm{pc}$. 
Fig. 4.- The same sample as in Figure 3 but with a disk-clearing model superimposed. The solid line shows the flux expected from binary-disk systems if they clear gaps in their disks from 0.2 to 3 times the binary separation; the dashed line shows clearing from 0.4 to 1.8 times the binary separation. The model shown has $T_{1}=150 \mathrm{~K}, q=0.6, M_{d}=0.05 M_{\odot}$, $R_{d}=100 \mathrm{AU}$, and is the average over a range of inclination angles.

Fig. 5.- The effect of different disk parameters on gap clearing models for binary-disk systems. For each plot, one model parameter is varied while the others are held fixed. Unless otherwise specified, the model parameters are $T_{1}=150 \mathrm{~K}, q=0.6, M_{d}=0.01 M_{\odot}$, $R_{d}=100 \mathrm{AU}$, and $\lambda=1300 \mu \mathrm{m}$; all models are averaged over a range of inclination angles. Line types are as in Figure 4. (a) Varying disk temperature normalization $T_{1}$. (b) Varying temperature exponent $q$. (c) Varying disk mass $M_{d}$. (d) Varying wavelength of calculated flux. Note that the vertical scale is not the same on all plots.

Fig. 6.- IRAS $60 \mu \mathrm{m}$ flux vs. projected binary separation for pre-main-sequence binaries. Filled squares are detections, open squares are upper limits, and a " $\mathrm{T}$ " indicates that a system is part of a triple. Most binaries are detected, indicating the presence of circumstellar disks in most systems. There is no evidence for a relationship between $60 \mu \mathrm{m}$ flux and binary separation. 